\title{
Reduced Order Feedback Synthesis for Viscous Incompressible Flows *
}

\author{
Kazufumi Ito and Jeffry D. Schroeter ${ }^{\dagger}$
}

March 21, 1999

\begin{abstract}
We discuss the application of the reduced basis method for the simulation and control of unsteady viscous flows governed by the incompressible Navier-Stokes equations. We describe how to use this method in terms of the construction of a lower-order compensator design. Our approach includes a construction method of the optimal state feedback law for finite dimensional nonlinear regulator problems. The method is applied to construct a feedback law for the reduced order control model of the NavierStokes equations, and then we apply our feedback law to the original control system. Our method is demonstrated on a control problem formulated in a channel flow using a boundary velocity control. We also show how these ideas can be extended to control problems governed by partial differential equations. Numerical results are reported for the open and closed loop controls, and a compensator design is proposed to complete the closed loop dynamics.
\end{abstract}

Keywords: flow control, compensator design, Navier-Stokes equations, reduced basis method, finite element method.

\section{Introduction}

In this paper we discuss the application of a reduced order model for the simulation and control of unsteady viscous flows. The reduced order model is developed using the reduced basis method with Lagrange interpolation. We will use the reduced basis method for the construction of a lower order compensator design. Our discussion will focus on the active control of the incompressible Navier-Stokes equations, but the procedure can be applied to other control problems governed by partial differential equations.

Active control of partial differential equations in real time requires extensive computational time and resources. Using standard methods such as finite differences or

${ }^{*}$ This work was supported in part by the Air Force Office of Scientific Research under grant AFOSR F49620-95-0236 and the Office of Naval Research under grant ONR N00014-96-1-0265.

${ }^{\dagger}$ Center for Research in Scientific Computation, Department of Mathematics, North Carolina State University, Raleigh, NC 27695-8205 (kito@eos.ncsu.edu and jdschroe@eos.ncsu.edu). 
finite elements often result in problems that are too large to solve in a reasonable time. We develop a reduced order model which overcomes this difficulty. The reduced basis method uses basis functions which are generated from the equations being solved. Since the basis functions are problem dependent, the dynamics of the original system can effectively be captured while using a small number of basis functions. This results in a dense, low order system which can be solved efficiently. This is in contrast to finite element methods, which use piecewise polynomials as basis functions, resulting in a large, sparse system of equations.

In $\S 2$ we will discuss the simulation of the incompressible Navier-Stokes equations using the finite element method. In $\S 3$ we describe the reduced basis method. This includes the selection of the reduced basis subspace and the construction of the reduced order model for the time dependent Navier-Stokes equations. We will compare two techniques for the selection of basis functions, and compare the reduced order solutions with the full solution computed in $\S 2$. In $\S 4$ we discuss the reduced order optimal control problem and formulate the solution method for the nonlinear regulator problem. In $\S 5$ we describe the construction of the feedback synthesis based on the reduced order model, and we also develop a compensator design based on the reduced order control system and the constructed feedback synthesis.

\section{Simulation of the Navier-Stokes Equations}

The Navier-Stokes equations which govern viscous incompressible flow are

$$
\begin{aligned}
\frac{\partial \mathbf{u}}{\partial t}-\frac{1}{R e} \Delta \mathbf{u}+\mathbf{u} \cdot \nabla \mathbf{u}+\nabla p & =\mathbf{f} \quad \text { in } \Omega \times(0, T] \\
\nabla \cdot \mathbf{u} & =0 \quad \text { in } \Omega \times(0, T] \\
\mathbf{u} & =\mathbf{u}_{b} \quad \text { on } \Gamma \times[0, T] \\
\mathbf{u}(\mathbf{x}, 0) & =\mathbf{u}_{0}(\mathbf{x}) \quad \text { in } \Omega
\end{aligned}
$$

where $\mathbf{u}(\mathbf{x}, t)$ is the velocity, $p(\mathbf{x}, t)$ is the pressure, $\mathbf{f}(\mathbf{x}, t)$ is the body force, $R e$ is the Reynolds number, $\mathbf{u}_{b}$ is the boundary velocity, $\mathbf{u}_{0}(\mathbf{x})$ is the initial velocity, and $\Omega$ is a bounded region in $\mathbb{R}^{2}$ with boundary $\Gamma$.

We will cast (2.1)-(2.4) in a variational form and use a mixed finite element approximation. First, we will introduce some standard notations. We denote by $L^{2}(\Omega)$ the collection of square integrable functions defined on $\Omega$, and we define the inner product on $L^{2}(\Omega)$ by

$$
(\phi, \psi)=\int_{\Omega} \phi \psi d x
$$

Let

$$
\begin{aligned}
& H^{1}(\Omega)=\left\{u \in L^{2}(\Omega): \frac{\partial u}{\partial x_{i}} \in L^{2}(\Omega), i=1,2\right\} \\
& H_{0}^{1}(\Omega)=\left\{u \in H^{1}(\Omega):\left.u\right|_{\partial \Omega}=0\right\}
\end{aligned}
$$

The vector-valued counterparts of these spaces are denoted by bold face symbols, for example $\mathbf{H}^{1}(\Omega)=H^{1}(\Omega) \times H^{1}(\Omega)$.

The variational form of (2.1)-(2.4) is obtained by taking the $L^{2}$-inner product of Equation (2.1) with any function $\mathbf{v} \in \mathbf{H}_{0}^{1}(\Omega)$, and the $L^{2}$-inner product of Equation 
(2.2) with any function $q \in L^{2}(\Omega)$. Applying the divergence theorem we arrive at the following variational form:

$$
\begin{gathered}
\left(\mathbf{u}_{t}, \mathbf{v}\right)+\frac{1}{R e} a(\mathbf{u}, \mathbf{v})+b(\mathbf{u}, \mathbf{u}, \mathbf{v})-c(\mathbf{v}, p)=(\mathbf{f}, \mathbf{v}) \quad \text { for all } \mathbf{v} \in \mathbf{H}_{0}^{1}(\Omega) \\
c(\mathbf{u}, q)=0 \quad \text { for all } q \in L^{2}(\Omega)
\end{gathered}
$$

where $(\cdot, \cdot)$ denotes the $L^{2}$-inner product and we define the standard bilinear and trilinear forms as follows:

$$
\begin{aligned}
a(\mathbf{u}, \mathbf{v}) & =\int_{\Omega} \nabla \mathbf{u} \cdot \nabla \mathbf{v} d x \quad \text { for all } \mathbf{u}, \mathbf{v} \in \mathbf{H}^{1}(\Omega) \\
c(\mathbf{u}, q) & =\int_{\Omega}(\nabla \cdot \mathbf{u}) q d x \quad \text { for all } \mathbf{u} \in \mathbf{H}^{1}(\Omega), q \in L^{2}(\Omega) \\
b(\mathbf{u}, \mathbf{v}, \mathbf{w}) & =\frac{1}{2} \int_{\Omega}((\mathbf{u} \cdot \nabla \mathbf{v}) \cdot \mathbf{w}-(\mathbf{u} \cdot \nabla \mathbf{w}) \cdot \mathbf{v}) d x \quad \text { for all } \mathbf{u}, \mathbf{v}, \mathbf{w} \in \mathbf{H}^{1}(\Omega)
\end{aligned}
$$

where we use the skew-symmetric form of the trilinear form introduced in [1] so that the discretized equations preserve the skew symmetry property of the Navier-Stokes equations. We also have the following properties of these linear forms. All of the forms $a(\cdot, \cdot), b(\cdot, \cdot, \cdot)$, and $c(\cdot, \cdot)$ are continuous. The bilinear form $a(\cdot, \cdot)$ satisfies the following coercive property:

$$
a(\mathbf{u}, \mathbf{u}) \geq C_{1}\|\mathbf{u}\|_{1}^{2} \quad \text { for all } \mathbf{u} \in \mathbf{H}_{0}^{1}(\Omega) .
$$

The bilinear form $c(\cdot, \cdot)$ satisfies the following inf-sup condition, as explained in [2]: there exists $\gamma>0$ such that

$$
\inf _{q \in L^{2}(\Omega)} \sup _{\mathbf{v} \in \mathbf{H}_{0}^{1}(\Omega)}\left(\frac{c(\mathbf{v}, q)}{\|\mathbf{v}\|_{1}\|q\|_{0}}\right) \geq \gamma
$$

We will use the mixed finite element method to approximate Equations (2.5)-(2.6). That is, we seek solutions $\mathbf{u}^{h} \in \mathbf{V}^{h}$ and $p^{h} \in S^{h}$ such that

$$
\begin{gathered}
\left(\mathbf{u}_{t}^{h}, \mathbf{v}^{h}\right)+\frac{1}{R e} a\left(\mathbf{u}^{h}, \mathbf{v}^{h}\right)+b\left(\mathbf{u}^{h}, \mathbf{u}^{h}, \mathbf{v}^{h}\right)-c\left(\mathbf{v}^{h}, p^{h}\right)=\left(\mathbf{f}, \mathbf{v}^{h}\right) \text { for all } \mathbf{v}^{h} \in \mathbf{V}_{0}^{h} \\
c\left(\mathbf{u}^{h}, q^{h}\right)=0 \quad \text { for all } q^{h} \in S^{h}
\end{gathered}
$$

where $\mathbf{V}_{0}^{h} \subset \mathbf{H}_{0}^{1}(\Omega)$ and $S^{h} \subset L^{2}(\Omega)$ are approximating finite dimensional subspaces. We use piecewise quadratic and piecewise linear basis functions for the velocity and pressure, respectively. This selection, referred to as the Taylor-Hood element pair, is known to satisfy the condition (2.7).

The resulting approximating system can be written as the following system of equations:

$$
\begin{gathered}
Q \dot{u}+H u+B(u, u)+\left(\begin{array}{c}
B_{1}^{T} p \\
B_{2}^{T} p
\end{array}\right)=f \\
\left(\begin{array}{ll}
B_{1} & B_{2}
\end{array}\right)\left(\begin{array}{l}
u_{1} \\
u_{2}
\end{array}\right)=0
\end{gathered}
$$

where $Q$ is the mass matrix, $H$ is the stiffness matrix, $\left(\begin{array}{ll}B_{1} & B_{2}\end{array}\right)$ are the continuity matrices, and $B(u, u)$ is the quadratic form that approximates the convective term. To 
solve this system, we will project Equation (2.10) onto the divergence free subspace, as described in [3], by using the following projection preconditioner

$$
P=P_{L^{2}}(-\Delta)^{-1} P_{L^{2}},
$$

where

$$
P_{L^{2}}=I-\left(\begin{array}{c}
B_{1}^{T} \\
B_{2}^{T}
\end{array}\right)\left(B_{1} B_{1}^{T}+B_{2} B_{2}^{T}\right)^{-1}\left(B_{1} B_{2}\right)
$$

is the $L^{2}$-projection onto the divergence free space. Applying the preconditioner to (2.10)-(2.11) we have the following nonlinear system of equations

$$
P Q \dot{u}+P H u+P B(u, u)=P f .
$$

We used the Implicit Midpoint rule to integrate Equation (2.12) in time, ie

$$
\begin{gathered}
P Q \frac{u^{k+\frac{1}{2}}-u^{k}}{\Delta t / 2}+P H u^{k+\frac{1}{2}}+P B\left(u^{k+\frac{1}{2}}, u^{k+\frac{1}{2}}\right)=P f, \\
u^{k+1}=2 u^{k+\frac{1}{2}}-u^{k} .
\end{gathered}
$$

We solved (2.13)-(2.14) using Newton's method; the Newton step here is carried out using the iterative method GMRES. In Figure 1, we see the solution to these equations on the forward facing channel flow. The height of the inflow boundary is 3 , and that of the outflow boundary is 2 . For boundary conditions, we assume a parabolic velocity profile at the inflow and outflow, and zero boundary conditions on all other walls. The inflow condition is $\mathbf{u}(y)=\frac{1}{9} y(3-y)$ and the outflow condition is $\mathbf{u}(y)=\frac{3}{8}(3-y)(y-1)$. The equations (2.13)-(2.14) were solved using $\Delta t=0.1$ to a final time of $T=40$. Since

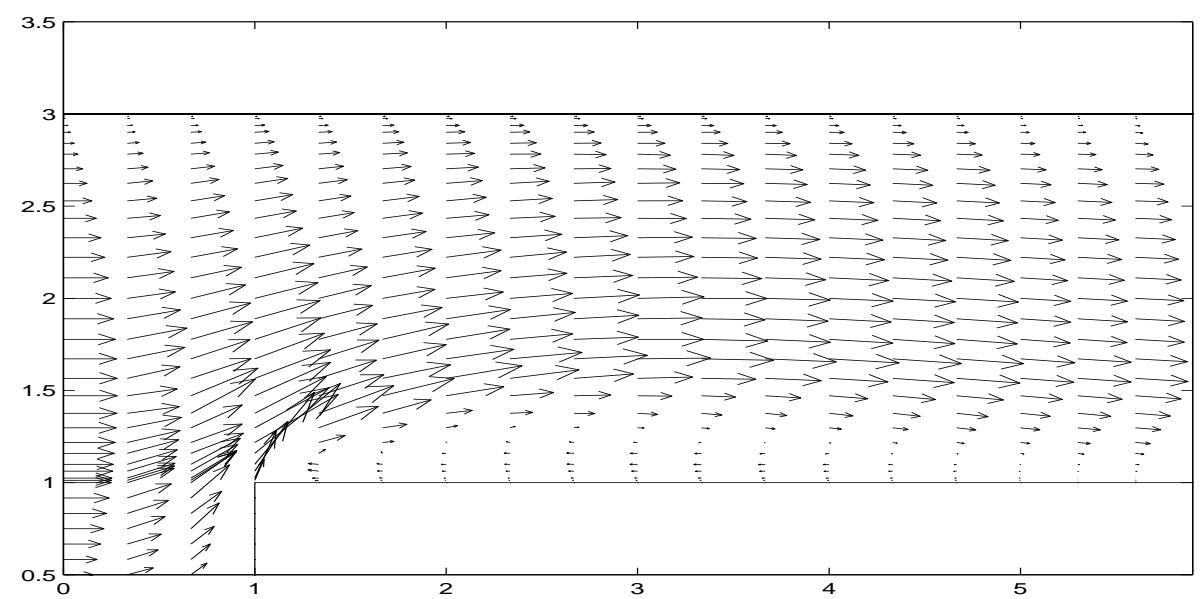

Figure 1: Time Dependent Navier-Stokes Equations at $T=40$

the initial condition must also satisfy the incompressibility condition (2.9), we used the solution to the stationary Stokes equations as the initial condition. The Stokes equations are the linear equations of the form

$$
\mathbf{u}_{t}-\Delta \mathbf{u}+\nabla p=0
$$




$$
\nabla \cdot \mathbf{u}=0
$$

Typically, a large number of unknowns is required to capture the complex flow patterns at high Reynolds numbers. For our computations, over 5000 unknowns were used. Using an Ultra Sun Sparc Station, the above computation required approximately 20 hours of computational time. We would like to develop a method to reduce the order of the system, thus requiring less computational time to solve, while still capturing the dynamics of the original system. We will describe this reduced order method in the next section.

\section{The Reduced Basis Method}

In this section we will present a reduced order method based on the reduced basis method. We would like this reduced order method to have the following characteristics:

1. Require a small number of basis functions.

2. Be inexpensive to solve.

3. Capture the dynamics of the original system.

The reduced basis method was first proposed in [4,5] for structural analysis, and it has been used for structural problems in $[6,7]$, and for incompressible flow problems in $[8,9]$. We will discuss the choice of reduced basis subspace and the construction of the reduced order equation. We will then develop a reduced order model for the incompressible Navier-Stokes equations, and show that it can effectively be used to capture unsteady viscous flows.

Consider the equation

$$
\mathcal{E}(u, \lambda)=0 \quad \text { for } u \in X, \lambda \in \Lambda
$$

where $\lambda$ represents a physical parameter, such as Reynolds number, and $u$ is a solution in a Hilbert space $X$. We will construct the reduced basis subspace $X^{R} \subset X$ by interpolation of the solution function $u(\lambda)$ of Equation (3.1) using one of the following three choices.

1. The Taylor Subspace In this case the reduced basis functions are linear combinations of Taylor basis functions generated by computing the Taylor expansion of $u(\lambda)$ at some reference value of $\lambda$, say $\lambda^{*}$. Let $u_{0}$ be the solution at the parameter $\lambda^{*}$, and assume it has $M$ derivatives. The reduced basis subspace is

$$
X^{R}=\operatorname{span}\left\{u_{j}: u_{j}=\left.\frac{\partial^{j} u}{\partial \lambda^{j}}\right|_{\lambda=\lambda^{*}}, j=0, \ldots, M\right\}
$$

The equations for $\frac{\partial^{j} u}{\partial \lambda^{j}}$ can be obtained by successive differentiation of Equation (3.1) with respect to the parameter $\lambda$. For example, $u_{1}=\frac{\partial u}{\partial \lambda}$ satisfies the linear equation

$$
\mathcal{E}_{u}\left(u_{0}, \lambda^{*}\right) u_{1}=-\mathcal{E}_{\lambda}\left(u_{0}, \lambda^{*}\right)
$$

Note that each subsequent $u_{j}, j=1, \ldots, M$ can be computed by solving a linear system with the same linear operator $E_{u}\left(u_{0}, \lambda^{*}\right)$. This choice has been proven effective for steady state fluid flow calculations, see [8]. However, one disadvantage is that if the parameter $\lambda$ moves away from the reference parameter $\lambda^{*}$, then the basis functions 
may have to be updated.

2. The Lagrange Subspace In this case the reduced basis functions are linear combinations of basis functions generated by solving the nonlinear system (3.1) at various parameter values $\lambda_{j}$. The reduced basis subspace is

$$
X^{R}=\operatorname{span}\left\{u_{j}: u_{j}=u\left(\lambda^{j}\right), j=1, \ldots, M\right\}
$$

An advantage with this choice is that the subspace can be updated by adding more basis functions, rather than updating the whole subspace.

3. The Hermite Subspace In this case the reduced basis functions are computed by solving the nonlinear system (3.1) at various parameter values $\lambda^{j}$, and computing the first derivatives at various parameter values. The reduced basis subspace is

$$
X^{R}=\operatorname{span}\left\{u_{j}=u\left(\lambda_{j}\right) \text { and }\left.\frac{\partial u}{\partial \lambda}\right|_{\lambda=\lambda_{j}}, j=1, \ldots, \hat{M}\right\}
$$

We can think of this choice as a hybrid of the Lagrange and Taylor approaches.

Let $\left\{\phi_{i}\right\}$ be a basis for the reduced basis subspace $X^{R} \subset X$, and let $m=\operatorname{dim}\left(X^{R}\right)$. If we let the reduced order solution be $u^{R}=\sum_{i=1}^{m} \alpha_{i} \phi_{i}$, then we obtain the reduced order problem by projecting Equation (3.1) onto the reduced basis space by the Galerkin approximation

$$
E^{R}\left(u^{R}, \lambda\right)_{i}=\left\langle E\left(u^{R}, \lambda\right), \phi_{i}\right\rangle_{X^{*} \times X}, i=1, \ldots, m .
$$

For the evolution equation

$$
\frac{d}{d t} u(t)+E(u(t), \lambda)=0
$$

we can generate the basis functions using solutions to the stationary problem as described previously, or we can use solutions to (3.2) at different time instances. The reduced order solution is $u^{R}(t)=\sum_{i=1}^{m} \alpha_{i}(t) \phi_{i}$, and the reduced order problem is

$$
\left\langle\frac{d}{d t} u^{R}(t)+E\left(u^{R}(t), \lambda\right), \phi_{i}\right\rangle_{X^{*} \times X}=0, i=1, \ldots, m
$$

We performed a comparison of the Lagrange and Hermite basis functions for the stationary Navier-Stokes equations. Using the approach described above, ie computing solutions at various Reynolds numbers, we were able to obtain a good approximation using 3 to 5 basis functions. The performance of the Lagrange versus the Hermite basis functions is comparable. Since the Lagrange basis functions require less time to compute and are also more adaptable, we will use Lagrange basis functions for the rest of our computations.

\subsection{Reduced Order Model for Viscous Flows}

In this section we will develop a reduced order model for the unsteady incompressible Navier-Stokes equations. The reduced basis functions will be generated using two different techniques:

1. Solutions to the time dependent Navier-Stokes equations at various time instances,

2. Solutions to the stationary Navier-Stokes equations with varying boundary velocity control force. 
In both cases we will use Lagrange interpolation to generate the reduced basis subspace. The former method is the more standard approach and has been proven effective in [10]. We use the latter approach because we feel it leads more naturally to the reduced order control problem. Through a comparison we will show that both techniques of basis generation produce a reduced order model which accurately captures the dynamics of the time dependent Navier-Stokes equations.

We will first illustrate the generation of the reduced basis functions from the time dependent Navier-Stokes equations. Define the reduced basis subspace as

$$
\mathbf{V}^{R}=\operatorname{span}\left\{\mathbf{u}_{i}: \mathbf{u}_{i}=\mathbf{u}^{h}\left(t_{i}\right), i=0, \ldots, m\right\}
$$

where $\mathbf{u}^{h}\left(t_{i}\right)$ is the finite element approximation at time $t_{i}$ to

$$
\begin{aligned}
\left(\mathbf{u}_{t}^{h}, \mathbf{v}^{h}\right)+\frac{1}{R e} a\left(\mathbf{u}^{h}, \mathbf{v}^{h}\right)+b\left(\mathbf{u}^{h}, \mathbf{u}^{h}, \mathbf{v}^{h}\right)-c\left(\mathbf{v}^{h}, p^{h}\right) & =\left(\mathbf{f}, \mathbf{v}^{h}\right) \quad \text { for all } \mathbf{v}^{h} \in \mathbf{V}_{0}^{h} \\
c\left(\mathbf{u}^{h}, q^{h}\right) & =0 \text { for all } q^{h} \in S^{h} \\
\left(\mathbf{u}^{h}, \mathbf{v}^{h}\right)_{\Gamma} & =\left(\mathbf{u}_{b}, \mathbf{v}^{h}\right)_{\Gamma} \text { for all } \mathbf{v}^{h} \in \mathbf{V}_{0}^{h} \\
\left(\mathbf{u}(x, 0), \mathbf{v}^{h}\right) & =\left(\mathbf{u}_{0}(x), \mathbf{v}^{h}\right) \quad \text { for all } \mathbf{v}^{h} \in \mathbf{V}_{0}^{h} .
\end{aligned}
$$

Define the reduced order solution as

$$
\mathbf{u}^{R}(t)=\phi_{0}+\sum_{i=1}^{m} \alpha_{i}(t) \phi_{i},
$$

where $\phi_{0}=\mathbf{u}_{t_{m}}^{h}$ is the solution at the final time. The basis functions $\left\{\phi_{i}\right\}_{i=1}^{m}$ are defined as

$$
\phi_{i}=\mathbf{u}^{h}\left(t_{i}\right)-\mathbf{u}^{h}\left(t_{i-1}\right), i=1, \ldots, m .
$$

Thus, $\phi_{0}$ has boundary conditions as the inflow/outflow conditions, $\left\{\phi_{i}\right\}_{i=1}^{m} \in \mathbf{V}_{0}^{R}=$ $\mathbf{V}^{R} \cap \mathbf{V}_{0}^{h}$ have homogeneous boundary conditions, and are used as test functions.

We will now illustrate the derivation of the reduced basis functions from the stationary Navier-Stokes equations. Define the reduced basis subspace as

$$
\mathbf{V}^{R}=\operatorname{span}\left\{\mathbf{u}_{i}=\mathbf{u}^{h}:\left.\mathbf{u}^{h}\right|_{\Gamma_{c}}=v_{i} \boldsymbol{\tau}, i=1, \ldots, m\right\}
$$

where the finite element approximation $\mathbf{u}^{h}$ satisfies

$$
\begin{aligned}
\frac{1}{R e} a\left(\mathbf{u}^{h}, \mathbf{v}^{h}\right)+b\left(\mathbf{u}^{h}, \mathbf{u}^{h}, \mathbf{v}^{h}\right)+c\left(\mathbf{v}^{h}, p^{h}\right) & =\left(\mathbf{f}, \mathbf{v}^{h}\right) \quad \text { for all } \mathbf{v}^{h} \in \mathbf{V}_{0}^{h} \\
c\left(\mathbf{u}^{h}, q^{h}\right) & =0 \quad \text { for all } q^{h} \in S^{h} \\
\left(\mathbf{u}^{h}, \mathbf{v}^{h}\right)_{\Gamma} & =\left(\mathbf{u}_{b}, \mathbf{v}^{h}\right)_{\Gamma} \text { for all } \mathbf{v}^{h} \in \mathbf{V}_{0}^{h} \\
\left.\mathbf{u}\right|_{\Gamma_{c}} & =v_{i} \boldsymbol{\tau}
\end{aligned}
$$

where $\Gamma_{c}$ is the control portion of the boundary, $v_{i}, i=1 \ldots, m$ are the boundary control velocities, and $\boldsymbol{\tau}$ is a unit tangential vector. Define the reduced order solution as

$$
\mathbf{u}^{R}(t)=\phi_{0}+\sum_{i=1}^{m} \alpha_{i}(t) \phi_{i}
$$

where $\phi_{0}=\mathbf{u}_{0}$ is the solution with zero boundary control velocity, and the basis functions $\left\{\phi_{i}\right\}_{i=1}^{m}$ are defined as

$$
\phi_{i}=\mathbf{u}_{m}-a_{i} \mathbf{u}_{i}+b_{i} \mathbf{u}_{0}, i=1, \ldots, m .
$$


The constants $a_{i}$ and $b_{i}$ are chosen to force homogeneous boundary conditions.

Once we have the reduced basis subspace $\mathbf{V}^{R}$, we can write the reduced order equation for the time dependent Navier-Stokes equations (2.1)-(2.4) as: Find $\mathbf{u}^{R}(t) \in$ $\mathbf{V}^{R}$ such that

$$
\begin{gathered}
\left(\mathbf{u}_{t}^{R}, \mathbf{v}^{R}\right)+\frac{1}{R e} a\left(\mathbf{u}^{R}, \mathbf{v}^{R}\right)+b\left(\mathbf{u}^{R}, \mathbf{u}^{R}, \mathbf{v}^{R}\right)=\left(\mathbf{f}, \mathbf{v}^{R}\right) \quad \text { for all } \mathbf{v}^{R} \in \mathbf{V}_{0}^{R} \\
\left(\mathbf{u}(x, 0), \mathbf{v}^{R}\right)=\left(\mathbf{u}_{0}(x), \mathbf{v}^{R}\right) \quad \text { for all } \mathbf{v}^{R} \in \mathbf{V}_{0}^{R}
\end{gathered}
$$

Note that the divergence free term does not appear since the reduced order basis functions satisfy the incompressibility condition (2.9). Substituting $\mathbf{u}^{R}=\phi_{0}+\sum_{i=1}^{m} \phi_{i}$ into Equation (3.3)-(3.4) with $\mathbf{v}^{R}=\phi_{j}, j=1, \ldots, m$, we obtain the following system for $\alpha_{i}(t), i=1, \ldots, m$ :

$$
\begin{gathered}
\left(\sum_{i=1}^{m} \dot{\alpha}_{i}(t) \phi_{i}, \phi_{j}\right)+\frac{1}{R e}\left(\nabla\left(\phi_{0}+\sum_{i=1}^{m} \alpha_{i}(t) \phi_{i}\right), \nabla \phi_{j}\right)+ \\
b\left(\phi_{0}+\sum_{i=1}^{m} \alpha_{i}(t) \phi_{i}, \phi_{0}+\sum_{i=1}^{m} \alpha_{i}(t) \phi_{i}, \phi_{j}\right)=\left(f, \phi_{j}\right), j=1, \ldots, m \\
\sum_{i=1}^{m} \alpha_{i}(0)\left(\phi_{i}, \phi_{j}\right)=\left(\mathbf{u}_{0}, \phi_{j}\right), j=1, \ldots, m
\end{gathered}
$$

We can write this system as the following system of nonlinear ordinary differential equations

$$
\begin{gathered}
\mathcal{M} \dot{\alpha}(t)+\mathcal{A} \xi(t)+\operatorname{col}\left[\xi(t)^{T} \mathcal{P}_{i} \xi(t)\right]_{i=1}^{m}=F(t) \\
\mathcal{M} \alpha(0)=\alpha_{0}
\end{gathered}
$$

where $\alpha=\operatorname{col}\left(\alpha_{1}, \ldots, \alpha_{m}\right) \in \mathbb{R}^{m}, \xi=(1 \alpha)^{T} \in \mathbb{R}^{m+1}$, and we define the mass matrix $\mathcal{M}=\left(\mathcal{M}_{i j}\right) \in \mathbb{R}^{m \times m}$, the stiffness matrix $\mathcal{A}=\left(\mathcal{A}_{i j}\right) \in \mathbb{R}^{m \times(m+1)}$, the forcing term $F=\left(F_{i}\right) \in \mathbb{R}^{m}$, and the initial condition $U^{0}=\left(U_{i}^{0}\right) \in \mathbb{R}^{m}$ as follows:

$$
\begin{aligned}
\mathcal{M}_{i j} & =\left(\phi_{i}, \phi_{j}\right), i, j=1, \ldots, m, \\
\mathcal{A}_{i j} & =\left(\nabla \phi_{i-1}, \nabla \phi_{j-1}\right), i=2, \ldots, m+1, j=1, \ldots, m+1, \\
F_{i}(t) & =\left(F(t), \phi_{i}\right), i=1, \ldots, m, \\
U_{i}^{0} & =\left(\mathbf{u}_{0}, \phi_{i}\right), i=1, \ldots, m .
\end{aligned}
$$

The matrices $\mathcal{P}_{i} \in \mathbb{R}^{(m+1) \times(m+1)}$ are defined by

$$
\left(\mathcal{P}_{i}\right)_{j k}=\left(\phi_{j-1} \cdot \nabla \phi_{k-1}, \phi_{i}\right), i=1, \ldots, m, j, k=1, \ldots, m+1 .
$$

If we define the matrix $\mathcal{N}$ as $\left(\xi^{T} \mathcal{N} \xi\right)_{i}=\xi^{T} \mathcal{P}_{i} \xi, i=1, \ldots, m$, then we can write Equation (3.5)-(3.6) as

$$
\begin{gathered}
\mathcal{M} \dot{\alpha}(t)+\mathcal{A} \xi(t)+\xi(t)^{T} \mathcal{N} \xi(t)=F(t) \\
\mathcal{M} \alpha(0)=U^{0}
\end{gathered}
$$

This is a dense, low-order system of nonlinear ordinary differential equations which we solved using the Implicit Midpoint rule

$$
\mathcal{M} \frac{\alpha^{k+\frac{1}{2}}-\alpha^{k}}{\Delta t / 2}+\mathcal{A} \xi^{k+\frac{1}{2}}+\left(\xi^{k+\frac{1}{2}}\right)^{T} \mathcal{N} \xi^{k+\frac{1}{2}}=F^{k+\frac{1}{2}},
$$




$$
\begin{gathered}
\alpha^{k+1}=2 \alpha^{k+\frac{1}{2}}-\alpha^{k}, \\
\mathcal{M} \alpha^{0}=U^{0} .
\end{gathered}
$$

We solved this quadratic system using Newton's method. Since the order of the system is small, the Newton step can be solved efficiently using a direct solver.

We have shown that a reduced order model can be developed which is inexpensive to solve. In the next section we will show that this model can accurately capture the original dynamics using a small number of basis functions.

\subsection{Computations of the Reduced Order Model}

We will demonstrate the performance of the reduced order model for the time dependent Navier-Stokes equations on the forward facing channel flow problem. As described in the previous section, we will compare two techniques for the selection of basis functions. The basis functions generated from the time dependent solution are shown in Table 1.

\begin{tabular}{|l||c|c|c|c|c|}
\hline Basis Function & $\mathbf{u}_{0}$ & $\mathbf{u}_{1}$ & $\mathbf{u}_{2}$ & $\mathbf{u}_{3}$ & $\mathbf{u}_{4}$ \\
\hline Number of time steps & 100 & 200 & 300 & 400 & 500 \\
\hline
\end{tabular}

Table 1: Basis Functions Generated from the Time Dependent Navier-Stokes Equations

The basis functions are defined as follows:

$$
\begin{aligned}
& \phi_{0}=\mathbf{u}_{4} \\
& \phi_{1}=\mathbf{u}_{1}-\mathbf{u}_{0} \\
& \phi_{2}=\mathbf{u}_{2}-\mathbf{u}_{1} \\
& \phi_{3}=\mathbf{u}_{3}-\mathbf{u}_{2} \\
& \phi_{4}=\mathbf{u}_{4}-\mathbf{u}_{3} .
\end{aligned}
$$

The basis functions generated from the stationary solution with corresponding boundary velocity are shown in Table 2 . The control region is taken to be the portion of the lower boundary from $x=1$ to $x=5$.

\begin{tabular}{|l||c|c|c|c|c|c|}
\hline Basis Function & $\mathbf{u}_{0}$ & $\mathbf{u}_{1}$ & $\mathbf{u}_{2}$ & $\mathbf{u}_{3}$ & $\mathbf{u}_{4}$ & $\mathbf{u}_{5}$ \\
\hline Boundary velocity & 0 & 0.05 & 0.1 & 0.15 & 0.2 & 0.25 \\
\hline
\end{tabular}

Table 2: Basis Functions Generated from the Stationary Navier-Stokes Equations

The basis functions are defined as

$$
\begin{aligned}
\phi_{0} & =\mathbf{u}_{0} \\
\phi_{1} & =\mathbf{u}_{5}-5 \mathbf{u}_{1}+4 \mathbf{u}_{0} \\
\phi_{2} & =\mathbf{u}_{5}-\frac{5}{2} \mathbf{u}_{2}+\frac{3}{2} \mathbf{u}_{0} \\
\phi_{3} & =\mathbf{u}_{5}-\frac{5}{3} \mathbf{u}_{3}+\frac{2}{3} \mathbf{u}_{0} \\
\phi_{4} & =\mathbf{u}_{5}-\frac{5}{4} \mathbf{u}_{4}+\frac{1}{4} \mathbf{u}_{0} .
\end{aligned}
$$


In Figure 2 we see the full solution at $T=25$. The solution was generated from the mixed finite element approximation described in Section 2. In Figure 3 we see the reduced order solution using basis functions generated from the stationary solution. In Figure 4 we see the reduced order solution using basis functions generated from the time dependent solution. Since we used the solution to the Stokes equations as an initial condition for the full solution, we will use the projection of the Stokes solution onto the reduced basis space as an initial condition for the reduced order model.

Comparing Figures 3 and 4 with Figure 2, we see that the reduced order models do accurately capture the dynamics of the full solution.

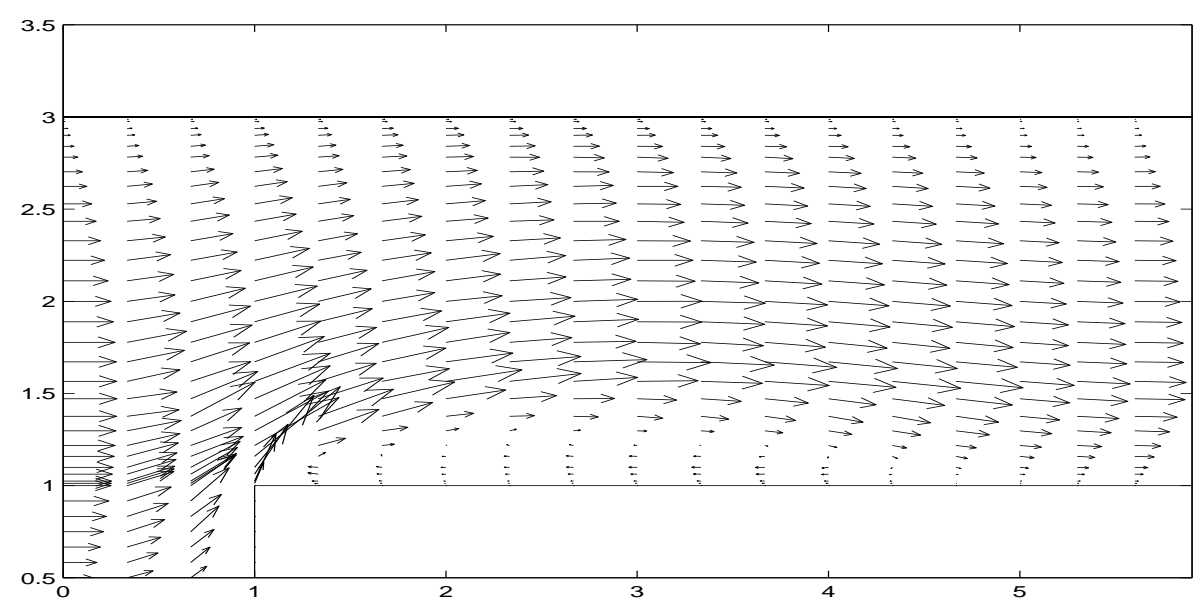

Figure 2: Full Solution at $\mathrm{T}=25$

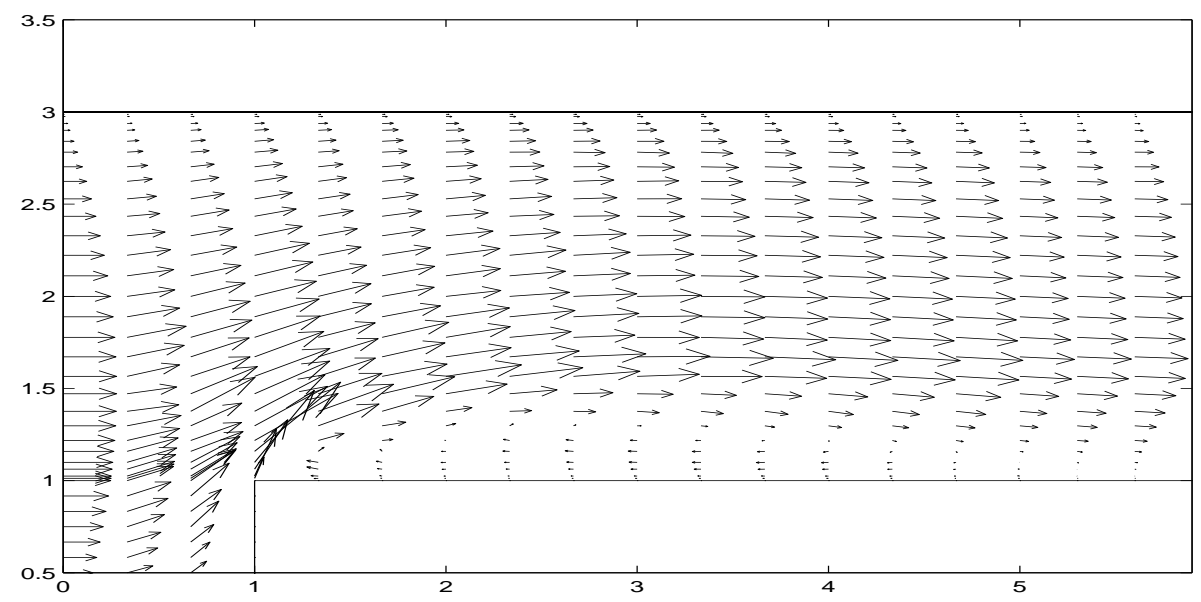

Figure 3: Reduced Order Solution with Stationary Basis Functions at $\mathrm{T}=25$

For a closer examination, in Figure 5 we see the velocity profiles of the reduced order models and the full solution at various time instances. The velocity profiles are chosen at $x=1,2,3,4,5$. These were chosen because this is where the recirculation is taking place, and it is this property which we want to capture with the reduced order models. 


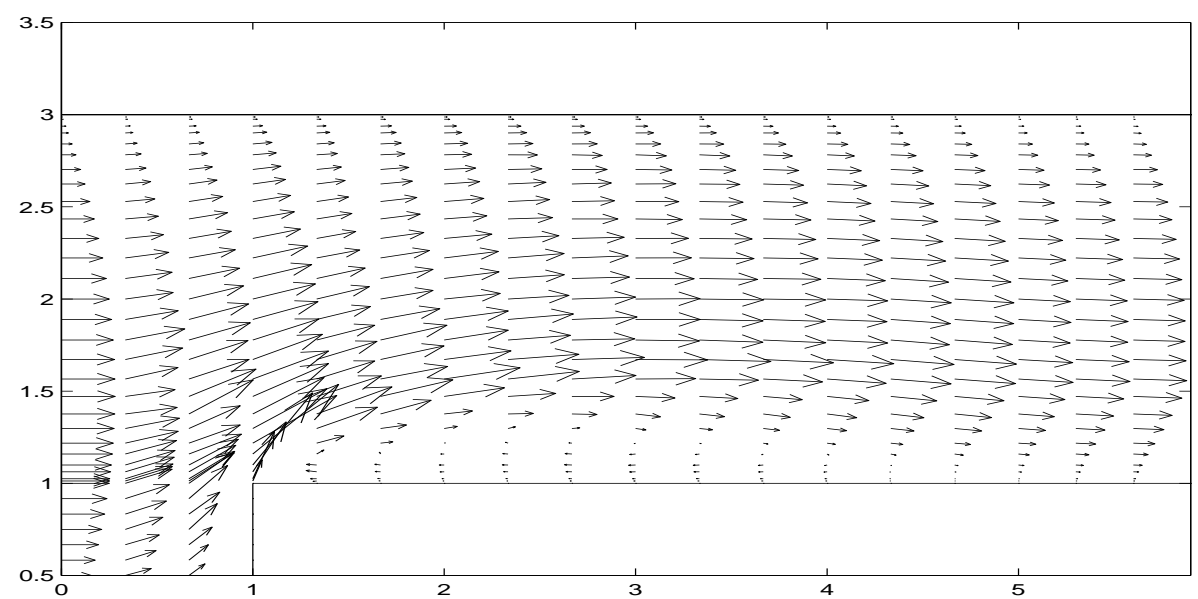

Figure 4: Reduced Order Solution with Time Dependent Basis Functions at $\mathrm{T}=25$

\section{Reduced Order Control Problem}

In this section we will develop a reduced order model for the control of the unsteady Navier-Stokes equations. From this reduced order system we will compute the necessary optimality conditions and outline a method of solution which can also be used to compute the open loop control for control problems governed by partial differential equations.

We begin with the development of the optimal control problem for viscous incompressible flows with boundary velocity control. Let us formulate the optimal control problem as

subject to

$$
\text { Minimize } \quad \mathcal{J}(\mathbf{u}, v(t))=\int_{0}^{T}\left(\mathcal{F}(\mathbf{u})+\frac{\beta}{2}|v(t)|^{2}\right) d t
$$

$$
\begin{aligned}
\left(\mathbf{u}_{t}, \mathbf{v}\right)+a(\mathbf{u}, \mathbf{v})+b(\mathbf{u}, \mathbf{u}, \mathbf{v})-c(\mathbf{v}, p) & =(\mathbf{f}, \mathbf{v}) \text { for all } \mathbf{v} \in \mathbf{H}_{0}^{1}(\Omega) \\
c(\mathbf{u}, q) & =0 \text { for all } q \in L^{2}(\Omega) \\
\mathbf{u} & =\mathbf{u}_{b} \text { on } \Gamma \\
\mathbf{u} & =u(t) \boldsymbol{\tau} \text { on } \Gamma_{c} \\
(\mathbf{u}(x, 0), \mathbf{v}) & =\left(\mathbf{u}_{0}(x), \mathbf{v}\right) \text { for all } \mathbf{v} \in \mathbf{H}_{0}^{1}(\Omega)
\end{aligned}
$$

where $\mathbf{f}$ is a fixed body force, $\mathbf{u}_{b}$ is a fixed boundary value on the boundary $\Gamma$. The function $u(t)$ is the control velocity defined on the control portion of the boundary $\Gamma_{c}$, and $\boldsymbol{\tau}$ is a unit tangential vector. In the cost functional, $\beta$ is the penalty term and $v(t)$ is the control force with the relation $\frac{d}{d t} u(t)=v(t)$. Note that by including $v(t)$ in the cost functional, we are minimizing the rate of change of the control velocity $u(t)$. The problem we will be studying is the minimization of vorticity in some subregion of the forward facing channel. We will use

$$
\mathcal{F}(\mathbf{u})=\mathcal{F}_{\text {vorticity }}=\int_{\Omega^{*}}|\nabla \times \mathbf{u}|^{2} d x
$$

where $\Omega^{*} \subset \Omega$ is the subregion where we wish to minimize vorticity. We will also use

$$
\mathcal{F}(\mathbf{u})=\mathcal{F}_{\text {tracking }}=\int_{\Omega^{*}}\left|\mathbf{u}-\mathbf{u}_{d}\right|^{2} d x
$$



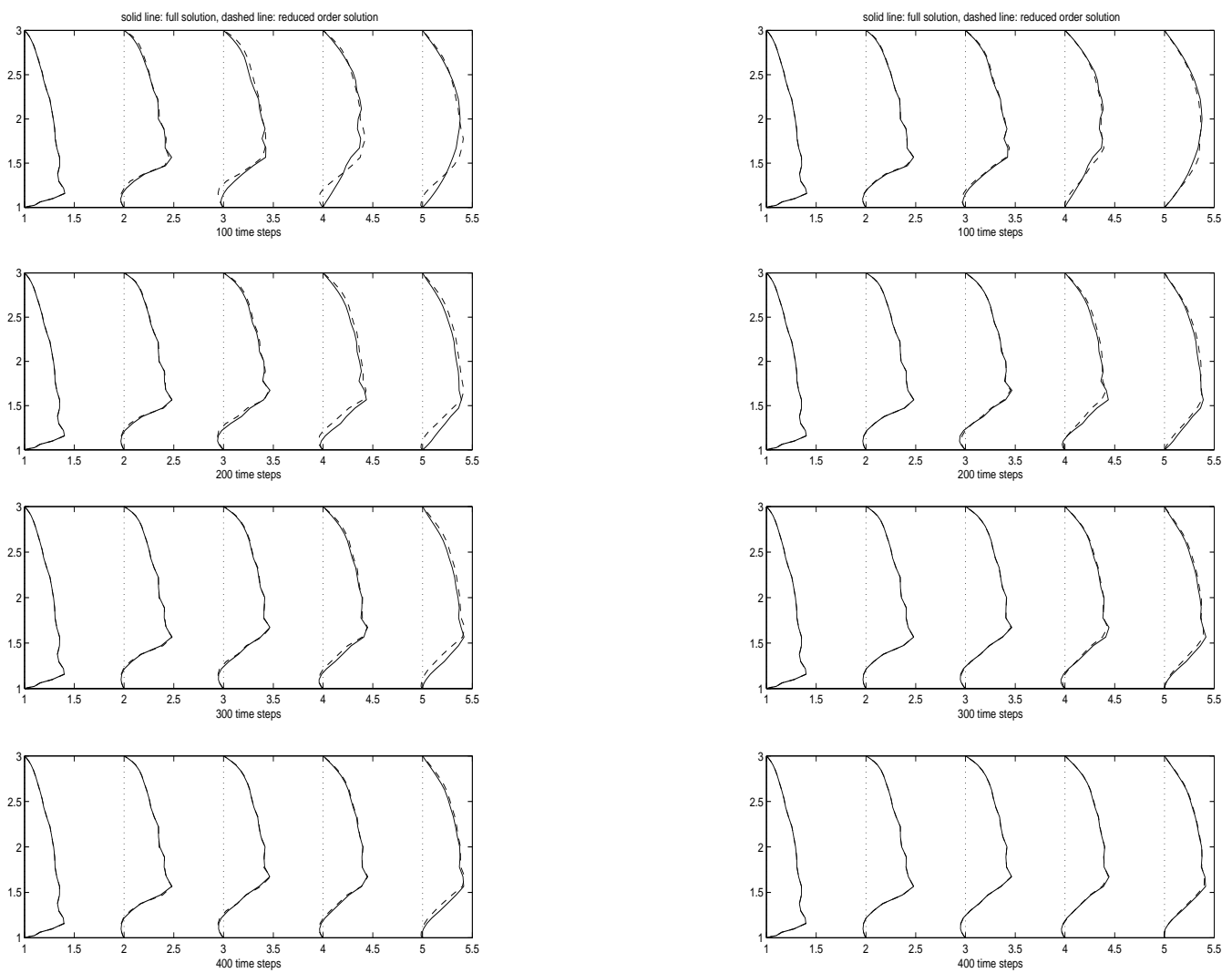

Basis functions from Stationary Solution Basis functions from Time Dependent Solution

Figure 5: Velocity Profiles for Forward Facing Channel Flow

where $\mathbf{u}_{d}$ is the solution to the Stokes equations. Since the Stokes equations do not have a recirculation region, we are minimizing vorticity by tracking to this solution.

We will now present the reduced order optimal control problem. The purpose of designing a reduced order model is so that the control can be computed in an efficient way, thus obtaining a suboptimal control law for the full system. Define the reduced order solution as

$$
x^{R}(t)=\phi_{0}+\sum_{i=1}^{m} \alpha_{i}(t) \phi_{i}+\sum_{j=1}^{n} u_{i}(t) \phi_{j+m}
$$

where $\phi_{0}$ corresponds to the stationary boundary force, ie the inflow/outflow conditions, $\left\{\phi_{i}\right\}_{i=1}^{m}$ are test functions with homogeneous boundary conditions, and $\left\{\phi_{j}\right\}_{j=m+1}^{m+n}$ are trial functions with corresponding boundary control force. The number of trial functions $n$ will be determined by the number of control inputs in the system. The number of test functions $m$ should be chosen large enough that they can accurately capture the dynamics of the system, yet small enough so that the reduced order system does not become ill-posed. For the forward facing channel flow problem with tangential boundary velocity control, we have one trial function corresponding to the control input, and we obtained good results using 2 to 4 test functions. 
We can write the reduced order controlled unsteady Navier-Stokes equations as

$$
\begin{gathered}
\mathcal{M} \dot{\alpha}(t)+\mathcal{A} x(t)+x(t)^{T} \mathcal{N} x(t)+b v(t)=0 \\
\dot{u}(t)=v(t) .
\end{gathered}
$$

We can now write the reduced order control problem as

$$
\text { Minimize } \quad \mathcal{J}(x, v)=\int_{0}^{T}\left(\frac{1}{2} x^{T} \mathcal{Q} x+\frac{\beta}{2}|v(t)|^{2}\right) d t
$$

subject to

$$
\begin{gathered}
\mathcal{M} \dot{\alpha}(t)+\mathcal{A} x(t)+x(t)^{T} \mathcal{N} x(t)+b v(t)=0 \\
\dot{u}(t)=v(t),
\end{gathered}
$$

where $\mathcal{Q}_{i j}=\left(\nabla \times \phi_{i}, \nabla \times \phi_{j}\right), i, j=1, \ldots, m$. Note that we can write this control problem in a general form as

$$
\text { Minimize } \quad \mathcal{J}(x(t), v(t))=\int_{0}^{T}(l(x(t))+h(v(t))) d t
$$

subject to

$$
\begin{aligned}
\dot{x}(t) & =f(x(t))+B v(t) \\
x(0) & =x_{0}
\end{aligned}
$$

where $x(t)=[\alpha(t) u(t)]^{T} \in \mathbb{R}^{m}$ is the state and $v(t)$ is the control. If we let $h(v(t))=$ $\frac{\beta}{2}|v(t)|^{2}$, then we can write the necessary conditions of optimality as

$$
\begin{aligned}
\dot{x}(t) & =f(x(t))+B v(t) \quad \text { a.e. in }(0, T), x(0)=x_{0}, \\
-\dot{p}(t) & =f_{x}(x(t))^{T} p(t)+l_{x}(x(t)) \quad \text { a.e. in }(0, T), p(T)=0, \\
v(t) & =-\frac{1}{\beta} B^{T} p(t) \quad \text { a.e. in }(0, T),
\end{aligned}
$$

which is the well known Pontryagin maximum principle, and $p(t) \in L^{2}(0, T)$ is the Lagrange multiplier. Thus the state-costate pair $(x(t), p(t))$ satisfies the two-point boundary value (TPBV) problem

$$
\begin{aligned}
\dot{x}(t) & =f(x(t))-\frac{1}{\beta} B B^{T} p(t) \\
-\dot{p}(t) & =f_{x}(x(t))^{T} p(t)+l_{x}(x(t))
\end{aligned}
$$

with $x(0)=x_{0}$ and $p(T)=0$, and the optimal control $v(t)$ for (4.9)-(4.11) is given by

$$
v(t)=\frac{-1}{\beta} B^{T} p(t) .
$$

In the next section we will develop a solution procedure for the TPBV problem (4.12)(4.13), and then present numerical results for the reduced order controlled NavierStokes equations. 


\subsection{Solution Procedure for Reduced Order Control Prob- lem}

In this section we will describe a general solution procedure to solve the TPBV problem (4.12)-(4.13). This method is described in [10] and is summarized here since the results are used directly in the design of the feedback synthesis.

We will discretize (4.9)-(4.11) using the Crank-Nicholson method for the dynamical constraint (4.10), and the trapezoidal rule for the cost functional (4.9), instead of directly discretizing the TPBV problem (4.12)-(4.13). That is,

$$
\text { Minimize } \quad \mathcal{J}^{N}=\sum_{k=1}^{N}\left(\frac{1}{2}\left(l\left(x^{k-1}\right)+l\left(x^{k}\right)\right)+h\left(v^{k}\right)\right) \Delta t
$$

subject to

$$
\frac{x^{k}-x^{k-1}}{\Delta t}=\frac{1}{2}\left(f\left(x^{k}\right)+f\left(x^{k-1}\right)\right)+B v^{k}, k=1, \ldots, N
$$

where $N \Delta t=T$ and $x^{0}=x_{0}$. The necessary optimality conditions for (4.14)-(4.15) are

$$
\begin{aligned}
\frac{x^{k}-x^{k-1}}{\Delta t} & =\frac{1}{2}\left(f\left(x^{k}\right)+f\left(x^{k-1}\right)\right)-\frac{1}{\beta} B B^{T} p^{k} \\
-\frac{p^{k+1}-p^{k}}{\Delta t} & =\frac{1}{2} f_{x}\left(x^{k}\right)^{T}\left(p^{k}+p^{k+1}\right)+l_{x}\left(x^{k}\right)
\end{aligned}
$$

for $k=1, \ldots, N$ and $x^{0}=x_{0}, p^{N+1}=0$, and the optimal control to (4.14)-(4.15) is given by

$$
v^{k}=-\frac{1}{\beta} B^{T} p^{k}
$$

As a consequence, (4.16)-(4.17) is an approximation to the TPBV problem (4.12)(4.13). System (4.16)-(4.17) is a sparse system of nonlinear equations in $x^{k}, p^{k}, k=$ $1, \ldots, N$, which can be solved using Newton's method:

$$
y_{+}=y_{c}-\left[F^{\prime}\left(y_{c}\right)\right]^{-1} F\left(y_{c}\right)
$$

where $y=\operatorname{col}\left(x^{1}, \ldots, x^{N}, p^{1}, \ldots, p^{N}\right)$, the function $F(y)=F\left(x^{1}, \ldots, x^{N}, p^{1}, \ldots, p^{N}\right)$ is given by

$$
F(y)=\left(\begin{array}{c}
\frac{x^{k}-x^{k-1}}{\Delta t}-\frac{1}{2}\left(f\left(x^{k}\right)+f\left(x^{k-1}\right)\right)+\frac{1}{\beta} B B^{T} p^{k} \\
\frac{p^{k+1}-p^{k}}{\Delta t}+\frac{1}{2} f_{x}\left(x^{k}\right)^{T}\left(p^{k}+p^{k+1}\right)+l_{x}\left(x^{k}\right)
\end{array}\right)
$$

and the Jacobian has the following matrix structure

$$
F^{\prime}=\left(\begin{array}{cc}
A & C \\
D & A^{T}
\end{array}\right)
$$

where $A$ is block lower bi-diagonal, and $C$ and $D$ are block diagonal with blocksize $m$. The diagonal and subdiagonal blocks of $A$ are given by

$$
A_{k, k}=\frac{I}{\Delta t}+\frac{1}{2} f_{x}\left(x^{k}\right) \quad \text { and } \quad A_{k+1, k}=-\frac{I}{\Delta t}+\frac{1}{2} f_{x}\left(x^{k}\right) .
$$


The diagonal block $C$ is the constant block

$$
C_{k, k}=\frac{1}{\beta} B B^{T},
$$

and the diagonal block $D$ is given by

$$
D_{k, k}=\frac{1}{2} f_{x x}\left(x^{k}\right)^{T}\left(p^{k}+p^{k+1}\right)+l_{x x}\left(x^{k}\right) .
$$

If we reorder the vector $y=\operatorname{col}\left(x^{1}, \ldots, x^{N}, p^{1}, \ldots, p^{N}\right)$ to $y=\operatorname{col}\left(x^{1}, p^{1}, x^{2}, p^{2}, \ldots, x^{N}, p^{N}\right)$ then the Jacobian is a block tridiagonal matrix with blocksize $2 m$. Thus the Newton step can be solved using a block tridiagonal algorithm.

\subsection{Computations of the Open Loop Control Problem}

We consider the control problem in a forward facing step channel flow. The goal is to minimize the recirculation that occurs at the top of the step by tangential movement of the boundary near the recirculation region, as seen in the schematic in Figure 6 .

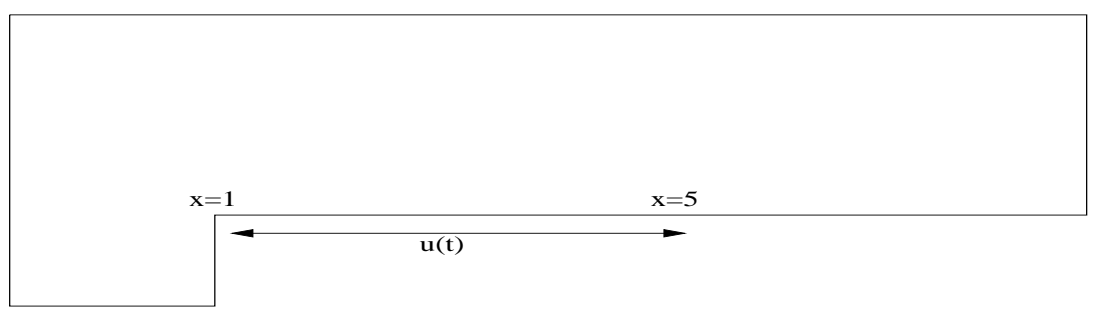

Figure 6: Schematic for Controlled Forward Facing Channel Flow

The basis functions $\left\{\mathbf{u}_{i}, i=0, \ldots, 5\right\}$ were chosen as solutions to the stationary NavierStokes equations with boundary control velocity as shown in Table 3.

\begin{tabular}{|l||c|c|c|c|c|c|}
\hline Basis Function & $\mathbf{u}_{0}$ & $\mathbf{u}_{1}$ & $\mathbf{u}_{2}$ & $\mathbf{u}_{3}$ & $\mathbf{u}_{4}$ & $\mathbf{u}_{5}$ \\
\hline Boundary Velocity & 0 & 0.05 & 0.1 & 0.15 & 0.2 & 0.25 \\
\hline
\end{tabular}

Table 3: Basis Functions Generated by Boundary Velocities

Define the reduced order solution as

$$
\mathbf{u}^{R}(t)=\phi_{0}+\sum_{i=1}^{4} \alpha_{i}(t) \phi_{i}+u(t) \phi_{5}
$$

where the basis functions are defined as

$$
\begin{aligned}
\phi_{0} & =\mathbf{u}_{0} \\
\phi_{1} & =\mathbf{u}_{5}-5 \mathbf{u}_{1}+4 \mathbf{u}_{0} \\
\phi_{2} & =\mathbf{u}_{5}-\frac{5}{2} \mathbf{u}_{2}+\frac{3}{2} \mathbf{u}_{0} \\
\phi_{3} & =\mathbf{u}_{5}-\frac{5}{3} \mathbf{u}_{3}+\frac{2}{3} \mathbf{u}_{0} \\
\phi_{4} & =\mathbf{u}_{5}-\frac{5}{4} \mathbf{u}_{4}+\frac{1}{4} \mathbf{u}_{0} \\
\phi_{5} & =4\left(\mathbf{u}_{5}-\mathbf{u}_{0}\right)
\end{aligned}
$$


Using the parameters $\Delta t=0.05, \beta=\frac{1}{50}$, and an initial condition $x_{0}=\left[\begin{array}{llll}0 & 0 & 0 & 0\end{array}\right]^{T}$, we obtained convergence of the two point boundary value problem in 9 iterations. The optimal control $u(t)$ is shown in Figure 7 . The flow simulation with the optimal control input is shown in Figure 8.

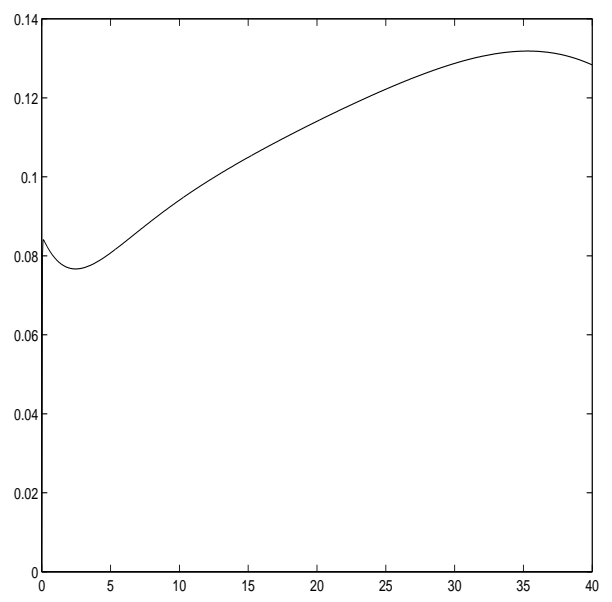

Figure 7: Control $u(t)$ for Forward Channel Flow

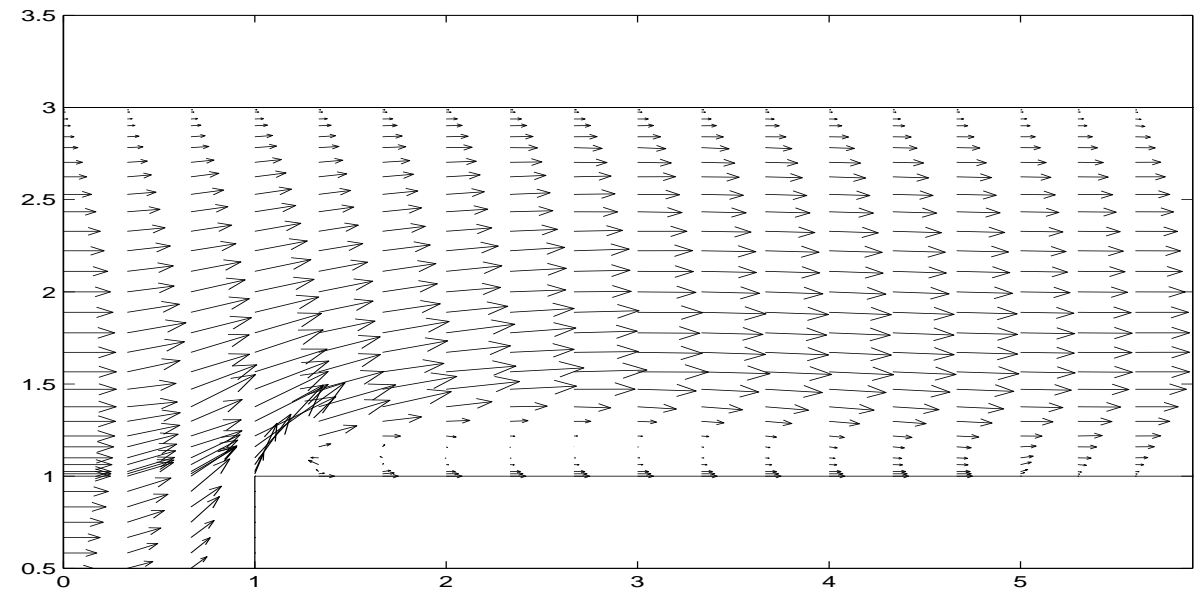

Figure 8: Navier-Stokes Equations with Control Input

\section{$5 \quad$ Design of Feedback Synthesis}

In this section we will discuss a method for the construction of a feedback synthesis $v(t)=K(x(t))$ based on the reduced order control system

$$
\begin{gathered}
\mathcal{M} \dot{\alpha}(t)+\mathcal{A} x(t)+x(t)^{T} \mathcal{N} x(t)+b v(t)=0 \\
\dot{u}(t)=v(t) \\
\alpha(0)=\alpha_{0},
\end{gathered}
$$


and then applied to the unsteady Navier-Stokes equations.

Consider the optimal control problem (4.9)-(4.11) in $\mathbb{R}^{m}$. Then the optimal control can alternatively be given by the feedback form

$$
v(t)=K(x(t), t)=\Psi\left(x(t), V_{x}(x(t), t)\right),
$$

where $\Psi(x(t), p(t))=v(t)$ minimizes the Hamiltonian

$$
p(t) \cdot(f(x)+B(x) V)+h(v(t)) .
$$

Here, $V$ is the unique viscosity solution to the Hamilton-Jacobi-Bellman (HJB) equation

$$
\begin{gathered}
V_{t}+f(x) \cdot V_{x}-h^{*}\left(-B^{T} V_{x}\right)+l(x)=0 \\
V(x, T)=0,
\end{gathered}
$$

where $B(x)=B$ and $h^{*}$ is the conjugate function of $h$. If we use $h=\frac{\beta}{2}|v(t)|^{2}$, then the conjugate function is $h^{*}=-\frac{1}{2 \beta}|p(t)|^{2}$. Thus, the optimal feedback solution is given by

$$
v(t)=-\frac{1}{\beta} B^{T} V_{x}(x(t), t)=K(x(t), t) .
$$

Assuming $V(x(t), t) \in C^{1}\left((0, T) ; \mathbb{R}^{n}\right)$, we have the following relationship between the HJB equation and the Pontryagin maximum principle

$$
p(t)=V_{x}(x(t), t),
$$

where the pair $(x(t), p(t))$ satisfies the TPBV problem (4.12)-(4.13) with $x(0)=x_{0}$ and $p(T)=0$.

Note that the feedback gain $K(x(t), t)=K_{T}(x(t), t)$ depends on the final time $T$. Since the problem under consideration is autonomous, we can write $K_{T}(x(t), t)=$ $K_{T-t}(x(t), 0)$. Given $T>0$, define the function

$$
x_{0} \rightarrow \Phi\left(x_{0}\right)=p^{x_{0}}(0),
$$

where $(x(t), p(t))^{x_{0}}$ is the solution to the TPBV problem with $x(0)=x_{0}$. Then we can define the feedback law

$$
K_{T}\left(x_{0}, 0\right)=\Psi\left(x_{0}, p^{x_{0}}(0)\right)=-\frac{1}{\beta} B^{T} \Phi\left(x_{0}\right)
$$

at each point $x_{0} \in \mathbb{R}^{m}$. Since the optimal feedback law $K_{T-t}(x(t), 0)$ is impractical, we replace the time dependent feedback law with the stationary feedback law

$$
v(t)=\bar{K}(x(t))=K_{T}(x(t), 0)
$$

for $T>0$ sufficiently large.

Based on these observations, we can construct a suboptimal feedback law by carrying out the following steps:

1. Choose a set of collocation points $\Sigma \subset \mathbb{R}^{m}$ and calculate the solution $(x(t), p(t))^{x_{0}}$ to the TPBV problem at the points $x_{0} \in \Sigma$. Thus we obtain the values of $\bar{K}$ at the points $x_{0} \in \Sigma$. 
2. Use an interpolation method to construct the feedback synthesis $K(x)$ satisfying

$$
K\left(x_{0}\right)=\bar{K}\left(x_{0}\right) \text { for } x_{0} \in \Sigma .
$$

For Step 2, we used an interpolation method based on the Green's function. Assume we have the set of collocation points $\left\{\left(x_{0}\right)_{i}\right\}_{i=1}^{M} \in \Sigma$. Define the feedback law

$$
K(x)=\sum_{i=1}^{M} G\left(x,\left(x_{0}\right)_{i}\right) \cdot \eta_{i}
$$

with

$$
G\left(\left(x_{0}\right)_{i},\left(x_{0}\right)_{j}\right) \cdot \eta_{j}=\bar{K}\left(\left(x_{0}\right)_{i}\right), i=1, \ldots, M .
$$

If the Green's functions $G$ satisfies the biharmonic equation

$$
\Delta^{2} G(x)=\delta(x),
$$

then $G(x)=|x|$ for $n=3$, and $G(x)=x^{2}(\ln x-1)$ for $n=2$. In this case, $K(x)$ minimizes

$$
\int_{\mathbb{R}^{n}}|\Delta K(x)|^{2} d x \quad \text { subject to } \quad K\left(\left(x_{0}\right)_{i}\right)=\bar{K}\left(\left(x_{0}\right)_{i}\right),\left(x_{0}\right)_{i} \in \Sigma .
$$

In our numerical calculations, we used a family of Green's functions of the form

$$
G(x, y)=|x-y|^{\alpha}, 1 \leq \alpha \leq 4 .
$$

\subsection{Computational Results of the Feedback Synthesis}

The design of the feedback law $K(x)$ requires that we compute the solution $(x(t), p(t))$ to the TPBV problem at a specified number of collocation points $\left(x_{0}\right)_{i}$. The extent of these points can be determined from the solution to the TPBV problem with the desired initial condition. In Figure 9 we show the reduced order solution $\left(\alpha_{1}(t), \alpha_{2}(t), u(t)\right)$ computed from the TPBV problem using the Stokes tracking problem (4.8) with an initial condition of $\left[\begin{array}{lll}0 & 0 & 0\end{array}\right]$. We chose the collocation points uniformly distributed on the intervals $[-2,2] \times[-15,15] \times[-0.5,0.5]$ for a total of $195=5 \times 13 \times 3$ points in the $\alpha_{1}, \alpha_{2}, u$ directions, respectively.

We used the Green's function $G(x, y)=|x-y|^{\alpha}$ with a value of $\alpha=3.7$. In Figure 10 we compare the interpolated values of $K(x)$ against the exact feedback law $\bar{K}(x)$. The parameter $u$ is fixed at $u=0.5$, and the values of $\bar{K}(x)$ are computed at 1281 uniformly distributed points in $([-2,2],[-15,15], 0.5) \in \mathbb{R}^{3}$. We see that this method does provide an accurate interpolant. In our calculations, we observed that lower values of $\alpha$ provided greater fluctuations in the interpolant.

In Figure 11 we compare the values of the open loop control and the closed loop control for the reduced order control system (5.18)-(5.20). It validates the feasibility of our approach.

We now describe how to apply the feedback control law on the full Navier-Stokes equations. To calculate the feedback control, we project the full solution onto the reduced basis space by

$$
P \mathbf{u}(t)=\phi_{0}+\sum_{i=1}^{2} \alpha_{i}(t) \phi_{i}+u(t) \phi_{3},
$$




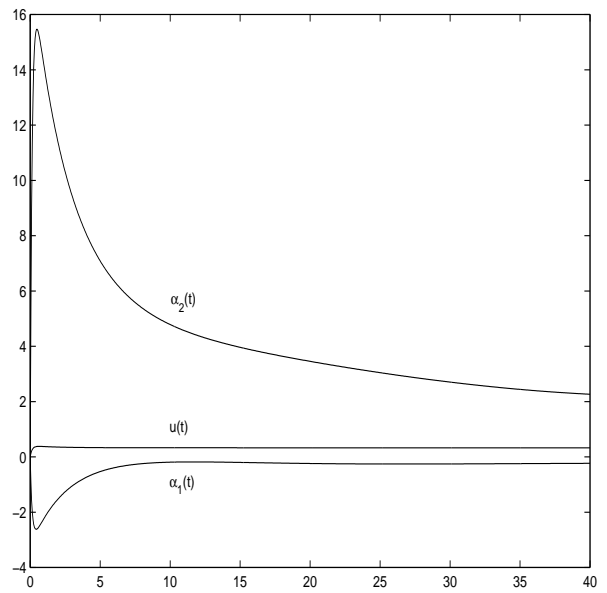

Figure 9: Reduced Order Parameters from Stokes Tracking Problem

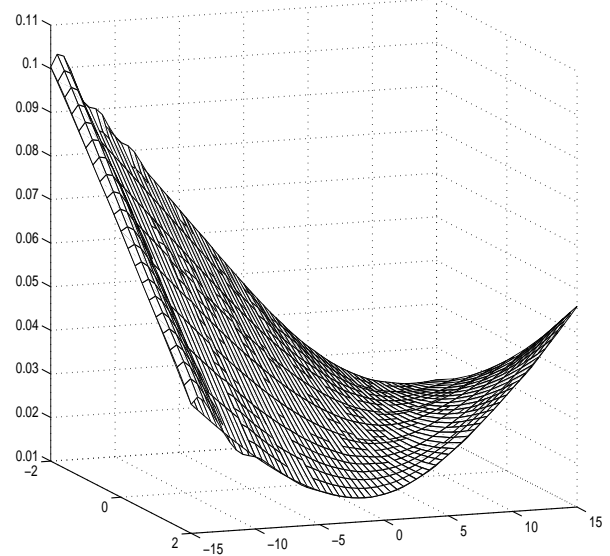

Figure 10: Interpolation of $B^{T} \Phi(x)$ with $u$ fixed at $0.5, \alpha=3.7$

where $\left(\alpha_{1}(t), \alpha_{2}(t)\right)$ is determined by the projection equation

$$
\left(\mathbf{u}(t), \phi_{j}\right)=\left(\phi_{0}+\sum_{i=1}^{2} \alpha_{i}(t) \phi_{i}+u(t) \phi_{3}, \phi_{j}\right), j=1,2 .
$$

Equation (5.21) is then written as

$$
\mathcal{M} \alpha=\operatorname{col}\left[\left(\mathbf{u}(t)-\phi_{0}-u(t) \phi_{m+1}, \phi_{j}\right)\right], j=1,2 .
$$

We can then construct the feedback law

$$
v(t)=K\left(\alpha_{1}(t), \alpha_{2}(t), u(t)\right),
$$

where $\left(\alpha_{1}(t), \alpha_{2}(t)\right)$ are computed from Equation (5.22). In Figure 12 we show the values of the closed loop control from the full Navier-Stokes equations compared with the values of the closed loop control computed from the reduced order model. The open loop control is also included as a reference. 


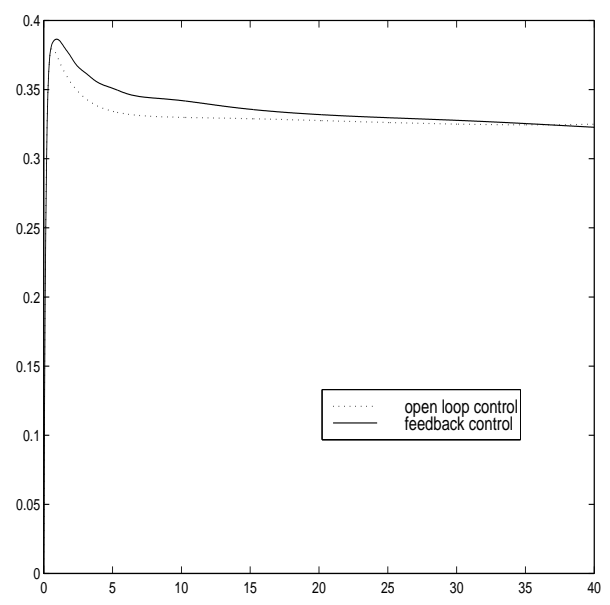

Figure 11: Feedback Control versus Open Loop Control

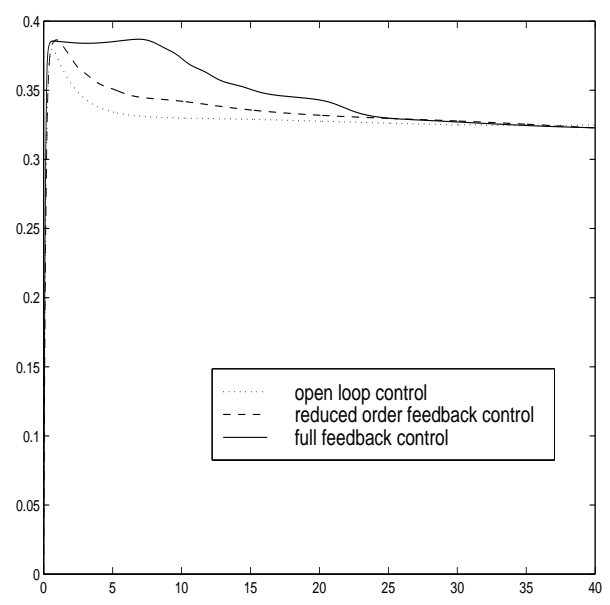

Figure 12: Feedback Control

\subsection{Dynamic Compensator Design}

The design of the feedback synthesis requires that we have a complete observation of the state at each time step. In practice, this is not feasible. For the full NavierStokes equations, rather than projecting the solution onto the reduced basis space and computing the feedback, we would like to be able to compute the feedback input $v(t)$ using a partial observation of the state. In this section, we propose a method of constructing a state estimator to complete the compensator dynamics.

The reduced order controlled Navier-Stokes equations can be written as

$$
\begin{gathered}
\frac{d x}{d t}+E(x, v)=0 \\
x(0)=x_{0},
\end{gathered}
$$

where $v=K(x)$ is the feedback control law and $x=\left[\begin{array}{ll}\alpha u\end{array}\right]^{T}$ are the parameters of the state. Assume we have a partial observation of the full Navier-Stokes equations

$$
y(t)=h(\mathbf{u}(t))
$$


where $h$ is a vector-valued, bounded, linear functional. We model the state estimator as

$$
\begin{aligned}
\frac{d \hat{x}}{d t}+E(\hat{x}, v) & =G(y-H \hat{x}) \\
\hat{x}(0) & =\hat{x}_{0},
\end{aligned}
$$

where $v=K(\hat{x})$ is the feedback control law, $\hat{x}$ is the state estimator, and $H_{j}=$ $h\left(\phi_{j}\right), j=1, \ldots, m$ is the matrix representation of the observation functional $h$ on $\mathbf{V}^{R}$. We compute the observer gain $G$ using the extended Kalman filter. Let $A=\frac{\partial}{\partial x} E(\hat{x}, v)$ be the linearized map at $\hat{x}$. Then the gain $G$ is given by

$$
G=\Sigma H^{T} R^{-1}
$$

where the error covariance $\Sigma$ is the solution to the Ricatti equation

$$
\frac{d}{d t} \Sigma=A \Sigma+\Sigma A-\Sigma H^{T} R^{-1} H \Sigma+Q
$$

and $Q, R$ are the covariance matrices of the system and observation noise, respectively.

\section{Conclusions}

We have presented a reduced order model based on the reduced basis method that can be used for the simulation and control of viscous incompressible flow problems. This reduced order model is inexpensive to solve and accurately captures the dynamics of the original system using a small number of basis functions. The feasibility of the reduced order model for flow control was demonstrated on a boundary velocity control problem. A low order feedback control law was developed and successfully implemented on the full Navier-Stokes equations. Through our numerical experiments, the feasibility of this feedback law was demonstrated for the control of viscous flows. With the state estimator, we have a complete compensator design for the full Navier-Stokes equations.

\section{References}

[1] R. Temam, Navier-Stokes Equations: Theory and Numerical Analysis, NorthHolland, Amsterdam, 1979.

[2] M. D. Gunzburger, Finite Element Methods for Viscous Incompressible Flows, Academic Press, London, 1989.

[3] A. J. Chorin and J. E. Marsden, A Mathematical Introduction to Fluid Mechanics, Springer-Verlag, New York, 1990.

[4] B. O. Almroth, P. Stern and F. A. Brogan, Automatic Choice of Global Shape Functions in Structural Analysis, AIAA J. 16, 1978.

[5] D. A. NAGy, Modal Representation of Geometrically Nonlinear Behavior by the Finite Element Method, Comput. \& Structures 10, 1979.

[6] A. K. Noor And J. M. Peters, Reduced Basis Technique for Collapse Analysis of Shells, AIAA J. 18, 1980. 
[7] A. K. Noor And J. M. Peters, Recent Advances in Reduction Methods for Instability Analysis of Structures, Comput. \& Structures 16, 1983.

[8] J. S. Peterson, The Reduced Basis Method for Incompressible Flow Calculations, SIAM J. Sci. Stat. Comput. 10, 1989.

[9] K. Ito And S. S. Ravindran, A Reduced Order Method for Simulation and Control of Fluid Flows, J. Comput. Phys. 143, 1998.

[10] K. Ito And S. S. Ravindran, Reduced Basis Method for Optimal Control of Unsteady Viscous Flows, Technical Report CRSC-TR97-28, Center for Research in Scientific Computation, North Carolina State University, 1997. 